\title{
GAIA Non-Reassuring Fetal Status Level of Diagnostic Certainty Terminology
}

National Cancer Institute

\section{Source}

National Cancer Institute. GAIA Non-Reassuring Fetal Status Level of Diagnostic

Certainty Terminology. NCI Thesaurus. Code C126857.

A subset of terminology related to non-reassuring fetal status, developed by the Global Alignment of Immunization safety Assessment in pregnancy consortium to aid in monitoring and improving fetal and maternal outcomes. 